\title{
Gender Specificity and Interpretation of Functional Cardiac Imaging: Let's Talk about Sex
}

\author{
Baris Gencer $^{1,2}$ François Mach ${ }^{1}$ \\ ${ }^{1}$ Cardiology Division, Geneva University Hospitals, Geneva, \\ Switzerland \\ 2 TIMI Study Group, Brigham and Women's Hospitals, Harvard Medical \\ School, Boston, Massachusetts, United States
}

Thromb Haemost 2019;119:1379-1381.

\section{Introduction}

In their present study, Fiechter et al report from nuclear medicine that for women, a strong association exists between inflammation, as measured by vertebral bone marrow metabolism, and impaired myocardial function and perfusion, as assessed by single photon emission computed tomography-myocardial perfusion imaging (SPECT-MPI). ${ }^{1}$ Among a total of 294 patients (28.6\% women, mean age 67 years old) who underwent ${ }^{18} \mathrm{~F}$-fluorodeoxyglucose $\left({ }^{18} \mathrm{~F}-\mathrm{FDG}\right)$ positron emission tomography and ${ }^{99} \mathrm{Tc}$-tetrofosmin SPECT-MPI, a significant increase in ${ }^{18} \mathrm{~F}-\mathrm{FDG}$ bone marrow uptake was observed in women with impaired myocardial perfusion as compared with normal myocardial perfusion (standardized uptake $2.2 \pm 1.2$ vs. $1.7 \pm 1.2$, $p=0.013)$, while no differences were found in men (1.6 \pm 0.8 vs. $1.6 \pm 0.4, p=0.372$ ). In addition, a significant inverse correlation between left ventricular ejection fraction and bone marrow metabolism was seen in women $(r=-0.229$, $p=0.037)$, but not in men $(r=-0.075, p=0.289)$. The present findings are relevant, interesting, and novel as no previous study has applied two different nuclear medicine imaging modalities simultaneously to investigate the degree of inflammation and the amount of myocardial ischemia perfusion in the same patient. In particular, a major difference between genders was found, suggesting the importance of the gender specificity when interpreting cardiac functional test.

\section{Gender Specificities in Cardiovascular Epidemiology}

It is well known that inflammation plays a fundamental role in mediating all phases of atherosclerosis, both in men and women. $^{2}$ Cardiovascular (CV) disease is still the leading cause of mortality for women globally, although the trend over the last years clearly shows a reduction of mortality
Address for correspondence François Mach, MD, FESC, Cardiology Division, Department of Specialties in Medicine, Geneva University Hospitals, Rue Gabrielle-Perret Gentil 4, 1211 Geneva 14, Switzerland (e-mail: Francois.mach@hcuge.ch).

both for men and women. ${ }^{3}$ Women are characterized by stronger immune responses and have a higher prevalence of inflammatory rheumatologic conditions compared with men. ${ }^{4}$ For instance, elevated high-sensitive C-reactive protein values were associated with a greater risk of stroke and hypertension among women compared with men. ${ }^{5}$ The recent guidelines highlighted the consideration of womenspecific risk factors in the history, including gestational diabetes and hypertension, preeclampsia, eclampsia, autoimmune disorders, obstructive sleep apnea, and radiationinduced myocardial injury. ${ }^{6}$ The 2016 European Society of Cardiology guidelines for prevention recommend starting systematic CV risk assessment in women from the age of 50 years or after menopause even in case of no known CV risk factor. Women are generally considered at a lower CV risk than men, with a gap of the estimated risk to be approximately 10 years (e.g., the risk of a 60-year-old woman is similar to that of a 50 -year-old man). ${ }^{7}$ However, the period following menopause is characterized by an increase of the CV risk profile and it is estimated that new coronary calcification occurs at a rate of $6 \%$ per year, as detected with the calcium score. $^{8}$

The need to define a prevention strategy tailored to address the women's CV risk associated with inflammation is becoming all the more pressing given the increased prevalence of active smoking and the additional detrimental effect of passive smoking in women compared with men. ${ }^{9}$ In addition, among middle-aged and older women, the prevalence of physical inactivity and obesity is substantially higher compared with men and associated with higher levels of inflammatory markers. ${ }^{10}$ Some strategies have shown to be more promising in women. For example, the measurement of the intima-media thickness, a subclinical marker of $\mathrm{CV}$ disease, seems to be more predictive of clinical CV events in women than in men. ${ }^{11}$ Also, efforts to prevent CV disease in women should probably start before the onset of the received

July 17, 2019

accepted

July 17,2019 (c) 2019 Georg Thieme Verlag KG Stuttgart · New York
DOI https://doi.org/ $10.1055 / \mathrm{s}-0039-1695010$. ISSN 0340-6245. 
menopause to be more beneficial. Overall, several health care interventions specifically adapted to women have already proved to be successful. ${ }^{6}$

\section{Gender Specificities in Coronary Artery Disease}

Women with established coronary artery disease (CAD) also have relevant specificities that need to be emphasized. ${ }^{6}$ Plaque anatomy and characteristics can differ between men and women, with a greater role of microvascular disease in the pathophysiology of coronary events among women ( - Fig. 1). ${ }^{6}$ At the time of manifestation of CV disease, women tend to be older, and with a greater risk factor profile and symptom burden of angina with positive ischemia on stress testing. ${ }^{6}$ However, positive ischemia on stress testing does not preclude having a higher prevalence of nontreatable lesions. Previous reports have suggested that women have paradoxically less obstructive coronary disease than men at the angiography exam. ${ }^{12}$ Along these lines, the clinical prognosis of symptomatic women with ischemia and nonobstructive CAD is worse than asymptomatic women. ${ }^{13}$ Therefore, the role of additional noninvasive evaluation in women with chest pain, including cardiac magnetic resonance stress testing for subendocardial ischemia or cardiac myocardial perfusion nuclear imaging for coronary flow reserve, are determinant to detect abnormalities due to microvascular ischemia. ${ }^{14}$

Another striking point is, unfortunately, the persisting disparity in clinical outcomes that still prevails between women and men known to have CAD., ${ }^{3,15}$ Observational data suggest that women admitted with myocardial infarction tend to receive reperfusion therapy and other evidence-based treatments less frequently and/or in a delayed manner compared with men. ${ }^{16,17}$ The presence of atypical symptoms is still more frequent in women, and the absence of chest pain is not a protective factor of CAD severity. ${ }^{18}$ Furthermore, evidence still points to the unfavorable outcomes associated with gender after myocardial infarction., ${ }^{3,19}$ Elevated residual inflammation predicts poor health status, and young women with acute myocardial infarction are known to have higher levels of inflammation compared with young men. ${ }^{20}$ In addition to

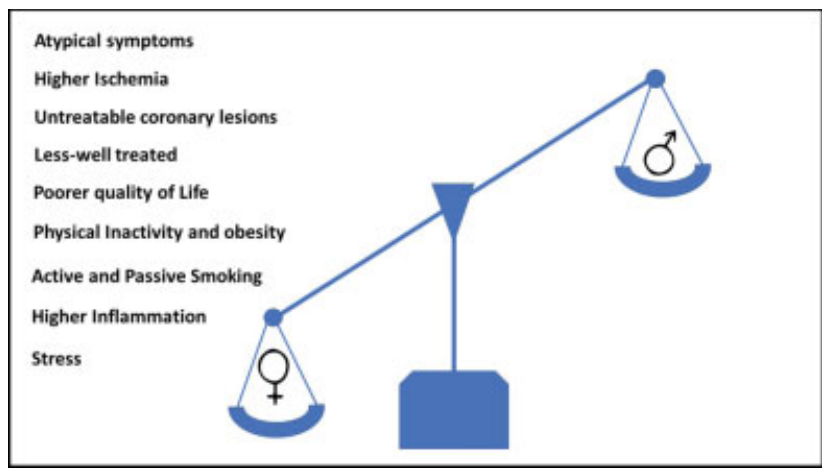

Fig. 1 Gender specificities for the prevention of cardiovascular disease. inflammation, higher levels of lipoprotein(a) have also been observed in women with CAD. ${ }^{21,22}$ Furthermore, quality of life is significantly impaired in women with CAD compared with men. ${ }^{23}$

\section{Conclusion}

The current study concurs with other evidence from the literature highlighting substantial gender disparities in several areas of CV imaging interpretation, prevention, and treatment. These gender disparities in the physiopathology mechanisms of atherosclerosis translate into higher $\mathrm{CV}$ mortality rates in women compared with men. Imaging modalities are appropriate screening and diagnostic tools to detect inflammation to improve CV prevention. This analysis is, to our knowledge, the first to combine two imaging diagnostic methods and show a relationship between increased vertebral bone marrow metabolic activity and impaired cardiac function and perfusion in women. These findings strengthen the role of inflammation in CAD and ischemic cardiomyopathy and the potential of imaging methods using the inflammatory process to improve prediction of CAD in women.

Future studies in the form of randomized controlled trials should clarify whether nuclear medicine can add prognostic value in medical decision-making and improve the prognosis of CAD in asymptomatic women. The sex differences in various CVD states have been well recognized. ${ }^{24}$ Integrating gender specificities in scientific statements, medical investigations, and practical guidelines will be determinant in improving women's health care..$^{25,26}$

\section{Conflict of Interest \\ None declared.}

\section{References}

1 Fiechter M, Haider A, Bengs S. Sex differences in the association between inflammation and ischemic heart disease. Thromb Haemost 2019;119(09):1471-1480

2 Spronk HMH, Padro T, Siland JE, et al. Atherothrombosis and thromboembolism: position paper from the Second Maastricht Consensus Conference on Thrombosis. Thromb Haemost 2018; 118(02):229-250

3 Mehta LS, Beckie TM, DeVon HA, et al; American Heart Association Cardiovascular Disease in Women and Special Populations Committee of the Council on Clinical Cardiology, Council on Epidemiology and Prevention, Council on Cardiovascular and Stroke Nursing, and Council on Quality of Care and Outcomes Research. Acute myocardial infarction in women: a scientific statement from the American Heart Association. Circulation 2016;133(09):916-947

4 Kurmann RD, Mankad R. Atherosclerotic heart disease in women with autoimmune rheumatologic inflammatory conditions. Can J Cardiol 2018;34(04):381-389

5 Jiménez MC, Rexrode KM, Kotler G, et al. Association between markers of inflammation and total stroke by hypertensive status among women. Am J Hypertens 2016;29(09):1117-1124

6 Raeisi-Giglou P, Volgman AS, Patel H, Campbell S, Villablanca A, Hsich E. Advances in cardiovascular health in women over the past decade: guideline recommendations for practice. J Womens Health (Larchmt) 2018;27(02):128-139 
7 Piepoli MF, Hoes AW, Agewall S, et al; ESC Scientific Document Group. 2016 European Guidelines on cardiovascular disease prevention in clinical practice: the Sixth Joint Task Force of the European Society of Cardiology and Other Societies on Cardiovascular Disease Prevention in Clinical Practice (constituted by representatives of 10 societies and by invited experts): developed with the special contribution of the European Association for Cardiovascular Prevention \& Rehabilitation (EACPR). Eur Heart J 2016;37(29):2315-2381

8 Kuller LH, Matthews KA, Edmundowicz D, Chang Y. Incident coronary artery calcium among postmenopausal women. Atherosclerosis 2008;200(02):278-285

9 Iversen B, Jacobsen BK, Løchen ML. Active and passive smoking and the risk of myocardial infarction in 24,968 men and women during 11 year of follow-up: the Tromsø Study. Eur J Epidemiol 2013;28(08):659-667

10 Lee IM, Sesso HD, Ridker PM, Mouton CP, Stefanick ML, Manson JE. Physical activity and inflammation in a multiethnic cohort of women. Med Sci Sports Exerc 2012;44(06):1088-1096

11 Chambless LE, Heiss G, Folsom AR, et al. Association of coronary heart disease incidence with carotid arterial wall thickness and major risk factors: the Atherosclerosis Risk in Communities (ARIC) Study, 1987-1993. Am J Epidemiol 1997;146(06):483-494

12 Nugent L, Mehta PK, Bairey Merz CN. Gender and microvascular angina. J Thromb Thrombolysis 2011;31(01):37-46

13 Gulati M, Cooper-DeHoff RM, McClure C, et al. Adverse cardiovascular outcomes in women with nonobstructive coronary artery disease: a report from the Women's Ischemia Syndrome Evaluation Study and the St James Women Take Heart Project. Arch Intern Med 2009;169(09):843-850

14 Mieres JH, Gulati M, Bairey Merz N, et al; American Heart Association Cardiac Imaging Committee of the Council on Clinical Cardiology; Cardiovascular Imaging and Intervention Committee of the Council on Cardiovascular Radiology and Intervention. Role of noninvasive testing in the clinical evaluation of women with suspected ischemic heart disease: a consensus statement from the American Heart Association. Circulation 2014;130(04): 350-379

15 Pilote L, Karp I. GENESIS-PRAXY (GENdEr and Sex determInantS of cardiovascular disease: From bench to beyond-Premature Acute Coronary SYndrome). Am Heart J 2012;163(05):741-746

16 Ibanez B, James S, Agewall S, et al; ESC Scientific Document Group. 2017 ESC Guidelines for the management of acute myocardial infarction in patients presenting with ST-segment elevation: the Task Force for the management of acute myocardial infarction in patients presenting with ST-segment elevation of the European Society of Cardiology (ESC). Eur Heart J 2018;39(02):119-177

17 Diercks DB, Owen KP, Kontos MC, et al. Gender differences in time to presentation for myocardial infarction before and after a national women's cardiovascular awareness campaign: a temporal analysis from the Can Rapid Risk Stratification of Unstable Angina Patients Suppress ADverse Outcomes with Early Implementation (CRUSADE) and the National Cardiovascular Data Registry Acute Coronary Treatment and Intervention Outcomes Network-Get with the Guidelines (NCDR ACTION RegistryGWTG). Am Heart J 2010;160(01):80-87.e3

18 Khan NA, Daskalopoulou SS, Karp I, et al; GENESIS PRAXY Team. Sex differences in acute coronary syndrome symptom presentation in young patients. JAMA Intern Med 2013;173(20):1863-1871

19 Pelletier R, Khan NA, Cox J, et al; GENESIS-PRAXY Investigators. Sex versus gender-related characteristics: which predicts outcome after acute coronary syndrome in the young? J Am Coll Cardiol 2016;67(02):127-135

20 Lu Y, Zhou S, Dreyer RP, et al. Sex differences in inflammatory markers and health status among young adults with acute myocardial infarction: results from the VIRGO (Variation in Recovery: Role of Gender on Outcomes of Young Acute Myocardial Infarction Patients) study. Circ Cardiovasc Qual Outcomes 2017; 10(02):e003470

21 Gencer B, Rigamonti F, Nanchen D, et al. Prognostic value of elevated lipoprotein(a) in patients with acute coronary syndromes. Eur J Clin Invest 2019;49(07):e13117

22 O'Donoghue ML, Fazio S, Giugliano RP, et al. Lipoprotein(a), PCSK9 inhibition, and cardiovascular risk. Circulation 2019;139(12): 1483-1492

23 Gencer B, Girardin F. Coronary artery disease is associated with persistent lower quality of life in women. Open Heart 2015;2(01): e000305

24 Cove CL, Albert CM, Andreotti F, Badimon L, Van Gelder IC, Hylek EM. Female sex as an independent risk factor for stroke in atrial fibrillation: possible mechanisms. Thromb Haemost 2014;111 (03):385-391

25 Linde C, Bongiorni MG, Birgersdotter-Green U, et al; ESC Scientific Document Group. Sex differences in cardiac arrhythmia: a consensus document of the European Heart Rhythm Association, endorsed by the Heart Rhythm Society and Asia Pacific Heart Rhythm Society. Europace 2018;20(10):1565-ao

26 Weber C, Badimon L, Mach F, van der Vorst EPC. Therapeutic strategies for atherosclerosis and atherothrombosis: past, present and future. Thromb Haemost 2017;117(07):1258-1264 